PROCEEDINGS OF THE

AMERICAN MATHEMATICAL SOCIETY

Volume 131, Number 9, Pages 2663-2671

S 0002-9939(03)06990-9

Article electronically published on April 1, 2003

\title{
ORTHOCOMPLETE EFFECT ALGEBRAS
}

\author{
GEJZA JENČA AND SYLVIA PULMANNOVÁ
}

(Communicated by Lance W. Small)

\begin{abstract}
We prove that for every orthocomplete effect algebra $E$ the center of $E$ forms a complete Boolean algebra. As a consequence, every orthocomplete atomic effect algebra is a direct product of irreducible ones.
\end{abstract}

\section{INTRODUCTION}

Effect algebras were introduced by Foulis and Bennett in their paper [5] for the study of logical foundations of quantum mechanics. Independently, Chovanec and Kôpka introduced essentially equivalent structures called D-posets (see [14]). Another equivalent structure was introduced by Giuntini and Greuling in [6]. For more information about effect algebras see [4.

The class of effect algebras is a common generalization of several classes of well-established algebraic structures, in particular orthomodular lattices and MValgebras.

In the present paper we prove that in an orthocomplete effect algebra $E$, the sums of all orthogonal families of central elements are central elements and that joins and meets of all families of central elements exist in $E$ and that they are central. For finite families, these results were proved in 8 . For countable families, see [11. As a consequence, an orthocomplete atomic effect algebra is a direct product of irreducible effect algebras. In addition, we prove that an effect algebra is $\kappa$-orthocomplete iff every chain of cardinality $\kappa$ has a supremum.

\section{EFFECT ALGEBRAS}

An effect algebra is a partial algebra $(E ; \oplus, 0,1)$ with a binary partial operation $\oplus$ and two nullary operations 0,1 satisfying the following conditions:

(E1) If $a \oplus b$ is defined, then $b \oplus a$ is defined and $a \oplus b=b \oplus a$.

(E2) If $a \oplus b$ and $(a \oplus b) \oplus c$ are defined, then $b \oplus c$ and $a \oplus(b \oplus c)$ are defined and $(a \oplus b) \oplus c=a \oplus(b \oplus c)$.

(E3) For every $a \in E$ there is a unique $a^{\prime} \in E$ such that $a \oplus a^{\prime}=1$.

(E4) If $a \oplus 1$ exists, then $a=0$.

Received by the editors April 3, 2002.

2000 Mathematics Subject Classification. Primary 06F05; Secondary 03G25, 81P10.

Key words and phrases. Effect algebra, central element, orthocomplete.

This research was supported by grant G-1/7625/20 of MS̆ SR, Slovakia and grant VEGA $2 / 7193 / 20$. 
In an effect algebra $E$, we write $a \leq b$ iff there is $c \in E$ such that $a \oplus c=b$. It is easy to check that $\leq$ is a partial order on $E$. In this partial order, 0 is the least and 1 is the greatest element of $E$. Moreover, it is possible to introduce a new partial operation $\ominus ; b \ominus a$ is defined iff $a \leq b$ and then $a \oplus(b \ominus a)=b$. It can be proved that $a \oplus b$ is defined iff $a \leq b^{\prime}$ iff $b \leq a^{\prime}$. Therefore, it is usual to denote the domain of $\oplus$ by $\perp$. We say that elements $a$ and $b$ in an effect algebra $E$ are orthogonal if $a \perp b$. In what follows, when we write $a \oplus b$ we mean that $a \oplus b$ is defined (i.e., $a \perp b$ ). Owing to associativity (E2), we may omit parentheses in $a_{1} \oplus a_{2} \oplus a_{3}$ and $a_{1} \oplus a_{2} \oplus \cdots \oplus a_{n}$, the latter term being defined by induction. We will say that the elements $a_{1}, \ldots, a_{n}$ are orthogonal if the element $a_{1} \oplus \cdots \oplus a_{n}$ exists in $L$. More generally, we say that $\left\{a_{\alpha}\right\}_{\alpha}$ is an orthogonal family if every finite subfamily is orthogonal.

An effect algebra need not be lattice-ordered. However, as proved in [19 and 8], the following relationship between $\wedge, \vee$ and $\oplus$ holds: if $a \vee b$ exists and $a \perp b$, then $a \wedge b$ exists and

$$
a \oplus b=(a \wedge b) \oplus(a \vee b) .
$$

Moreover, it is easy to check (see [1]) that, for every subset $B$ of an effect algebra such that $\vee B$ exists and for every $x \geq B$,

$$
x \ominus(\vee B)=\wedge\{x \ominus b: b \in B\} .
$$

Example 2.1. Let $\left(L ; \wedge, \vee,{ }^{\prime}, 0,1\right)$ be an orthomodular lattice. Write $a \oplus b=a \vee b$ iff $a \leq b^{\prime}$, otherwise let $a \oplus b$ be undefined. Then $(L, \oplus, 0,1)$ is an effect algebra. Effect algebras, which are associated with orthomodular lattices in this way, can be characterized as lattice-ordered effect algebras satisfying the implication

$$
a \perp b \Longrightarrow a \wedge b=0 .
$$

Example 2.2. An $M V$-algebra (cf. [2, 15]) is a commutative semigroup ( $M ; \oplus$, $\neg, 0$ ), satisfying identities $x \oplus 0=x, \neg \neg x=x, x \oplus \neg 0=\neg 0$ and

$$
x \oplus \neg(x \oplus \neg y)=y \oplus \neg(y \oplus \neg x) .
$$

There is a natural partial order in an MV-algebra, given by $y \leq x$ iff $x=x \oplus$ $\neg(x \oplus \neg y)$. Every MV-algebra $(M ; \oplus, \neg, 0)$ can be considered as an effect algebra $(M ; \oplus, 0, \neg 0)$ when we restrict the operation $\oplus$ to the domain $\perp=\{(x, y): x \leq \neg y\}$. Effect algebras, which are associated with MV-algebras, can be characterized as lattice-ordered effect algebras satisfying the implication

$$
a \wedge b=0 \Longrightarrow a \perp b .
$$

(Cf. [17.)

Example 2.3. Let $H$ be a Hilbert space, and let $S(H)$ denote the partially ordered group of all bounded self-adjoint linear operators on $H$. Put $E(H)=\{A \in S(H)$ : $0 \leq A \leq 1\}$; the elements of $E(H)$ are called effects. For $a, b \in E(H)$, write $a \oplus b=a+b$ iff $a+b \in E(H)$, otherwise let $a \oplus b$ be undefined. Then $(E(H) ; \oplus, 0,1)$ is an effect algebra. We remark that for $\operatorname{dim}(H) \geq 2, E(H)$ is not lattice-ordered.

Let $E_{1}, E_{2}$ be effect algebras. A map $\phi: E_{1} \mapsto E_{2}$ is called a morphism iff it satisfies the following condition:

(H1) $\phi(1)=1$ and if $a \perp b$, then $\phi(a) \perp \phi(b)$ and $\phi(a \oplus b)=\phi(a) \oplus \phi(b)$.

A morphism $\phi: E_{1} \mapsto E_{2}$ of effect algebras is called full iff the following condition is satisfied: 
(H2) If $\phi(a) \perp \phi(b)$ and $\phi(a) \oplus \phi(b) \in \phi(E)$, then there exist $a_{1}, b_{1} \in E_{1}$ such that $a_{1} \perp b_{1}, \phi(a)=\phi\left(a_{1}\right)$ and $\phi(b)=\phi\left(b_{1}\right)$.

A bijective, full morphism is called an isomorphism. A morphism $\phi$ is an isomorphism if it is bijective and $\phi^{-1}$ is also a morphism.

Let $E_{1}$ be an effect algebra. A subset $E_{2} \subseteq E_{1}$ is a subeffect algebra of $E_{1}$ iff $0,1 \in E_{2}, E_{2}$ is closed under the ' operation, and $a, b \in E_{2}$ with $a \perp b \Longrightarrow a \oplus b \in$ $E_{2}$.

Another possibility to create a substructure of an effect algebra $E$ is to restrict $\oplus$ to an interval

$$
[0, a]=\{x \in E: 0 \leq x \leq a\}
$$

as follows. For $x, y \in[0, a], x \oplus y$ is defined iff $x \oplus y$ exists in $E$ and $x \oplus y \in[0, a]$. We can then consider $[0, a]$ as an effect algebra, letting $a$ act as the unit element. In what follows, we denote such effect algebras by $[0, a]_{E}$.

Let $E$ be an effect algebra. A subset $I$ of $E$ is called an ideal of $E$ iff the following condition is satisfied:

$$
x, y \in I \text { and } x \perp y \Leftrightarrow x \oplus y \in I .
$$

\section{ORThocomplete EFFECT ALgeBRAS AND CENTRAL ELEMENTS}

In this section, we will prove that the center of an orthocomplete effect algebra is a complete Boolean algebra. This is a generalization of [8] and [11.

Let $E$ be an effect algebra. Suppose that there is an isomorphism $\phi: E \mapsto$ $E_{1} \times E_{2}$. For every such $\phi$, the elements $\phi^{-1}(1,0)$ and $\phi^{-1}(0,1)$ are called central elements of $E$. We write $C(E)$ for the set of all central elements of an effect algebra $E$. We say that an effect algebra $E$ is irreducible iff $C(E)=\{0,1\}$.

Recall that an element $a \in E$ is sharp if $a \wedge a^{\prime}=0$, and $a$ is principal if $b, c \leq a$, $b \perp c$ implies $b \oplus c \leq a$. It is easy to see that a principal element is sharp; the opposite implication need not be true, in general. Central elements can be intrinsically characterized by the following properties: (i) $c$ and $c^{\prime}$ are principal and (ii) every element $x \in E$ admits a decomposition $x=x_{1} \oplus x_{2}$ with $x_{1} \leq c$, $x_{2} \leq c^{\prime}$. It can be proved that this decomposition of $x$ is unique. In fact, $x_{1}=x \wedge c$, $x_{2}=x \wedge c^{\prime}$. Moreover, for every central element $a$, the map $x \mapsto a \wedge x$ is a full morphism, which maps $E$ onto $[0, a]_{E}$ (cf. [12]).

It was proved in 8 that the set of all central elements forms a sub-effect algebra of $E$, which is a Boolean algebra. Moreover, the joins and meets of elements of $C(E)$ exist in $E$ and coincide with their joins and meets in $C(E)$. If $a, b \in C(E)$ are orthogonal, we have $a \vee b=a \oplus b$ and $a \wedge b=0$.

Lemma 3.1. If $x, y \in E$ and $a \in C(E)$, then

$$
a \wedge(x \oplus y)=a \wedge x \oplus a \wedge y .
$$

Moreover, if $a, b \in C(E)$, and $x \in E$, then

$$
x \wedge(a \oplus b)=x \wedge a \oplus x \wedge b .
$$

Proof. Let $x \perp y, x, y$ in $E$, and $a \in C(E)$. Then $x \oplus y=x \wedge a \oplus x \wedge a^{\prime} \oplus y \wedge a \oplus y \wedge a^{\prime}=$ $(x \wedge a \oplus y \wedge a) \oplus\left(x \wedge a^{\prime} \oplus y \wedge a^{\prime}\right)$, where the first summand is under $a$, the second under $a^{\prime}$. Uniqueness of the decomposition of $x \oplus y$ then yields $(x \oplus y) \wedge a=x \wedge a \oplus y \wedge a$.

If $a, b \in C(E), a \perp b$ and $x \in E$, then $(a \oplus b) \wedge x=(a \oplus b) \wedge\left(x \wedge a \oplus x \wedge a^{\prime}\right)=$ $(a \vee b) \wedge(x \wedge a) \oplus(a \vee b) \wedge\left(x \wedge a^{\prime}\right)=a \wedge x \oplus b \wedge x$. 
For all $a \in C(E)$, the interval $[0, a]$ is an ideal of $E$. These ideals are called central ideals. By [3], a central ideal in an effect algebra $E$ can be characterized as an ideal $I$ satisfying the following conditions:

- $I=[0, a]$ for some $a \in E$.

- $I$ is a Riesz ideal, i.e., if $i \in I$ and $i \leq a \oplus b$, then there exist $i_{1}, i_{2} \in I$, such that $i_{1} \leq a, i_{2} \leq b, i \leq i_{1} \oplus i_{2}$.

Let $E$ be an effect algebra, and $\left\{a_{\alpha}\right\}_{\alpha}$ be an orthogonal family. We define $\oplus_{\alpha} a_{\alpha}:=\vee_{F} \oplus\left(a_{\alpha}: \alpha \in F\right)$, where the supremum goes over all finite subfamilies $F$ of $\alpha$ 's, if the supremum on the right-hand side exists.

We will say that an effect algebra $E$ is $m$-orthocomplete for an infinite cardinal $m$ if every orthogonal family of at most $m$ elements has an $\oplus$-sum in $L$. We say that an effect algebra $E$ is orthocomplete if it is $m$-orthocomplete for every cardinal $m$.

The following theorem is a generalization of [11 Lemma 3.3]. For analogues in orthomodular lattices see [10, in orthoalgebras [9], [18.

Theorem 3.2. Let $E$ be an effect algebra and let $m$ be a cardinal. The following are equivalent:

(1) $E$ is m-orthocomplete.

(2) Every chain of at most $m$ elements has a supremum.

Proof. The implication that (1) implies (2) was proved in 13. We have to prove that (2) implies (1). Assume that every chain of at most $m$ elements in $E$ has a supremum. Let $X$ be an orthogonal subset of $E$ and let $\operatorname{card}(X) \leq m$. We may assume that $X$ is infinite, and let $\gamma$ be the first ordinal with $\operatorname{card}(\gamma)=\operatorname{card}(X)$. We will prove that the $\oplus X$ exists and it is equal to $\vee \Sigma$, where

$$
\Sigma:=\left(\oplus\left(x_{\alpha}: \alpha<\beta\right): \beta<\gamma\right),
$$

$\left(x_{\alpha}: \alpha<\gamma\right)$ being an indexing of $X$ with $\gamma$. We proceed by induction by $\operatorname{card}(X)$. If $X$ is finite, there is nothing to prove. Let $X$ be infinite, $\operatorname{card}(X) \leq m$, and $\operatorname{card}(X)=\operatorname{card}(\gamma)$. The induction hypothesis is that for all orthogonal sets $Y$, $\operatorname{card}(Y)=\beta<\gamma, \oplus Y$ exists, and

$$
\oplus Y=\vee\left(\oplus\left(x_{\sigma}: \sigma<\nu\right): \nu<\beta\right) .
$$

Let $X=\left(x_{\alpha}: \alpha<\gamma\right)$ be an indexing as desired. By induction hypothesis, the chain

$$
\Sigma:=\left(\oplus\left(x_{\alpha}: \alpha<\beta\right): \beta<\gamma\right)
$$

exists in $E$. Since $\operatorname{card}(\Sigma)=\operatorname{card}(X) \leq m$, the supremum $s:=\vee \Sigma$ exists in $E$. Let $x_{\alpha_{1}}, \ldots, x_{\alpha_{n}}$ be an arbitrary finite sequence with $\alpha_{1}, \ldots, \alpha_{n}<\gamma$. Without loss of generality, we may assume that $\alpha_{1} \leq \alpha_{2} \leq \cdots \leq \alpha_{n}$. Then $\left(x_{\alpha_{1}}, \ldots, x_{\alpha_{n}}\right) \subseteq\left(x_{\alpha}\right.$ : $\left.\alpha<\alpha_{n}+1\right)$ and hence

$$
s \geq \oplus\left(x_{\alpha}: \alpha<\alpha_{n}+1\right) \geq \oplus\left(x_{\alpha_{1}}, \ldots, x_{\alpha_{n}}\right) .
$$

This proves that $s$ is an upper bound of all $\oplus\left(x_{\alpha}: \alpha \in F\right), F$ being a finite subset of the index set $(\alpha: \alpha<\gamma)$. To see that $s$ is the desired supremum, let $p$ be an upper bound of all $\oplus\left(x_{\alpha}: \alpha \in F\right)$, where $F$ is a finite subsets of $(\alpha: \alpha<\gamma)$. Then for all $\beta<\gamma, p$ is an upper bound of $\oplus\left(x_{\alpha}: \alpha<\beta\right)$. From this it follows that $p$ is an upper bound of $\Sigma$, hence $p \geq s$. 
Consequently, in every orthocomplete effect algebra, every chain has a supremum.

Lemma 3.3. Let $E$ be an orthocomplete effect algebra. Let $\left(a_{\alpha}: \alpha \in \Sigma\right) \subseteq E$ be an orthogonal family of central elements. Let $\left(x_{\alpha}: \alpha \in \Sigma\right)$ be a family of elements satisfying $x_{\alpha} \leq a_{\alpha}$, for all $\alpha \in \Sigma$. Then $\vee\left(x_{\alpha}: \alpha \in \Sigma\right)$ exists and equals $\oplus\left(x_{\alpha}: \alpha \in \Sigma\right)$.

Proof. Obviously, $\left(x_{\alpha}: \alpha \in \Sigma\right)$ is an orthogonal family, so that $\oplus\left(x_{\alpha}: \alpha \in \Sigma\right)$ exists in $E$ by orthocompleteness. Let $M$ be a finite subset of $\Sigma$, and let $y$ be any upper bound of $\left(x_{\alpha}: \alpha \in M\right)$. Then clearly $\forall \alpha \in M, x_{\alpha} \leq y \wedge a_{\alpha}$. Therefore

$$
\begin{aligned}
\oplus\left(x_{\alpha}: \alpha \in M\right) & \leq \oplus\left(y \wedge a_{\alpha}: \alpha \in M\right) \\
& =y \wedge\left(\oplus\left(a_{\alpha}: \alpha \in M\right)\right) \leq y .
\end{aligned}
$$

Thus, $\oplus\left(x_{\alpha}: \alpha \in M\right)$ is under every upper bound of $\left(x_{\alpha}: \alpha \in M\right)$, and we see that for every finite nonempty $M \subset \Sigma$, we have $\oplus\left(x_{\alpha}: \alpha \in M\right)=\vee\left(x_{\alpha}: \alpha \in M\right)$. This implies that

$$
\begin{aligned}
\oplus\left(x_{\alpha}: \alpha \in \Sigma\right) & =\vee_{F} \oplus\left(x_{\alpha}: \alpha \in F\right) \\
& =\vee_{F} \vee\left(x_{\alpha}: \alpha \in F\right) \\
& =\vee\left(x_{\alpha}: \alpha \in \Sigma\right),
\end{aligned}
$$

where $\vee_{F}$ runs over all finite subsets $F$ of $\Sigma$.

Theorem 3.4. Let $E$ be an orthocomplete effect algebra. Let $\left(a_{\alpha}: \alpha \in \Sigma\right)$ be an orthogonal family of central elements. Denote $a=\oplus\left(a_{\alpha}: \alpha \in \Sigma\right)$. Then a is central and

$$
[0, a]_{E} \simeq \prod_{\alpha \in \Sigma}\left[0, a_{\alpha}\right]_{E}
$$

Proof. Define a mapping $\phi:[0, a]_{E} \rightarrow \prod_{\alpha \in \Sigma}\left[0, a_{\alpha}\right]_{E}$ by $\phi(x)=\left(x \wedge a_{\alpha}\right)_{\alpha \in \Sigma}$. We shall prove that $\phi$ is an isomorphism.

To prove that $\phi$ is onto, let $\left(x_{\alpha}\right)_{\alpha \in \Sigma} \in \prod_{\alpha \in \Sigma}\left[0, a_{\alpha}\right]_{E}$. Observe that $\left(x_{\alpha}: \alpha \in \Sigma\right)$ is an orthogonal family and put $x=\oplus\left(x_{\alpha}: \alpha \in \Sigma\right)$. We will prove that $\phi(x)=$ $\left(x_{\alpha}\right)_{\alpha \in \Sigma}$.

We have

$$
\phi(x)=\left(x \wedge a_{\alpha}\right)_{\alpha \in \Sigma}=\left(\left(\oplus\left(x_{\alpha}: \alpha \in \Sigma\right)\right) \wedge a_{\alpha}\right)_{\alpha \in \Sigma} .
$$

Fix $\beta \in \Sigma$. By associativity of $\oplus$,

$$
\begin{aligned}
x \wedge a_{\beta} & =\left(\oplus\left(x_{\alpha}: \alpha \in \Sigma\right)\right) \wedge a_{\beta} \\
& =\left(x_{\beta} \oplus\left(\oplus\left(x_{\alpha}: \alpha \in \Sigma \backslash\{\beta\}\right)\right)\right) \wedge a_{\beta} .
\end{aligned}
$$

Since the family $\left(x_{\alpha}: \alpha \in \Sigma \backslash\{\beta\}\right)$ satisfies conditions of Lemma 3.3, we have

$$
\begin{aligned}
\left(x_{\beta}\right. & \left.\oplus\left(\oplus\left(x_{\alpha}: \alpha \in \Sigma \backslash\{\beta\}\right)\right)\right) \wedge a_{\beta} \\
& =\left(x_{\beta} \oplus\left(\vee\left(x_{\alpha}: \alpha \in \Sigma \backslash\{\beta\}\right)\right)\right) \wedge a_{\beta}
\end{aligned}
$$

and since $a_{\beta}$ is a central element,

$$
\begin{aligned}
& \left(x_{\beta} \oplus\left(\vee\left(x_{\alpha}: \alpha \in \Sigma \backslash\{\beta\}\right)\right)\right) \wedge a_{\beta}=x_{\beta} \wedge a_{\beta} \oplus\left(\vee\left(x_{\alpha}: \alpha \in \Sigma \backslash\{\beta\}\right) \wedge a_{\beta}\right) \\
& \quad=x_{\beta} \oplus\left(\vee\left(x_{\alpha}: \alpha \in \Sigma \backslash\{\beta\}\right) \wedge a_{\beta}\right) .
\end{aligned}
$$


Since, for all $\alpha \in \Sigma \backslash\{\beta\}, x_{\alpha} \wedge a_{\beta}=0$, we have

$$
\left(\vee\left(x_{\alpha}: \alpha \in \Sigma \backslash\{\beta\}\right) \wedge a_{\beta}\right)=0 .
$$

Thus, for all $\alpha \in \Sigma, x \wedge a_{\alpha}=x_{\alpha}$.

To prove that $\phi$ is one-to-one, it suffices to prove that, for all $x \in[0, a]$,

$$
x=\oplus\left(x \wedge a_{\alpha}: \alpha \in \Sigma\right) .
$$

As

$$
\begin{aligned}
& \oplus \quad\left(x \wedge a_{\alpha}: \alpha \in \Sigma\right)=\vee_{F}\left(\oplus\left(x \wedge a_{\alpha}: \alpha \in F\right)\right) \\
& =\vee_{F}\left(x \wedge\left(\oplus a_{\alpha}: \alpha \in F\right)\right),
\end{aligned}
$$

where we used (44) in the last equality, we see that $\oplus\left(x \wedge a_{\alpha}: \alpha \in \Sigma\right) \leq x$. Moreover, using (2),

$$
\begin{aligned}
x \ominus\left(\oplus\left(x \wedge a_{\alpha}: \alpha \in \Sigma\right)\right) & =x \ominus\left(\vee_{F}\left(\oplus x \wedge a_{\alpha}: \alpha \in F\right)\right) \\
& =x \ominus\left(\vee_{F}\left(x \wedge\left(\oplus\left(a_{\alpha}: \alpha \in F\right)\right)\right)\right) \\
& =\wedge_{F}\left(x \ominus\left(x \wedge \oplus\left(a_{\alpha}: \alpha \in F\right)\right)\right) .
\end{aligned}
$$

Since $\forall F, \oplus\left(a_{\alpha}: \alpha \in F\right)$ is central, we have

$$
\begin{aligned}
\left(x \ominus\left(x \wedge \oplus\left(a_{\alpha}: \alpha \in F\right)\right)\right) & =x \wedge\left(\oplus\left(a_{\alpha}: \alpha \in F\right)\right)^{\prime} \\
& =x \wedge\left(\vee\left(a_{\alpha}: \alpha \in F\right)\right)^{\prime} \\
& =x \wedge\left(\wedge\left(a_{\alpha}^{\prime}: \alpha \in F\right)\right) .
\end{aligned}
$$

Therefore

$$
\begin{aligned}
\wedge_{F}\left(x \ominus\left(x \wedge \oplus\left(a_{\alpha}: \alpha \in F\right)\right)\right) & =\wedge_{F} x \wedge\left(\wedge\left(a_{\alpha}^{\prime}: \alpha \in F\right)\right) \\
& \leq \wedge_{F} a \wedge\left(\wedge a_{\alpha}^{\prime}: \alpha \in F\right) \\
& =\wedge_{F}\left(a \ominus\left(\vee\left(a_{\alpha}: \alpha \in F\right)\right)^{\prime}\right) \\
& =a \ominus\left(\vee_{F} \vee\left(a_{\alpha}: \alpha \in F\right)\right) \\
& =0 .
\end{aligned}
$$

Hence, for all $x \in[0, a]_{E}, x=\oplus\left(x \wedge a_{\alpha}: \alpha \in \Sigma\right)$, and this implies that $\phi$ is one-to-one.

Let us prove that $[0, a]$ is an ideal. Obviously, $x \oplus y \in[0, a]$ implies $x \perp y$ and $x, y \in[0, a]$. To prove the opposite implication, assume that $x, y \in[0, a]$ and $x \perp y$. By the preceding paragraph, $x=\oplus\left(x \wedge a_{\alpha}: \alpha \in \Sigma\right), y=\oplus\left(y \wedge a_{\alpha}: \alpha \in \Sigma\right)$. Then

$$
\begin{aligned}
x \oplus y & =\left(\oplus\left(x \wedge a_{\alpha}: \alpha \in \Sigma\right)\right) \oplus\left(\oplus\left(y \wedge a_{\alpha}: \alpha \in \Sigma\right)\right) \\
& =\left(\oplus\left(x \wedge a_{\alpha} \oplus y \wedge a_{\alpha}\right): \alpha \in \Sigma\right) .
\end{aligned}
$$

Since $\forall \alpha, a_{\alpha}$ is central,

$$
x \wedge a_{\alpha} \oplus y \wedge a_{\alpha}=(x \oplus y) \wedge a_{\alpha} \leq a_{\alpha} .
$$

Using Lemma 3.3 ,

$$
\begin{aligned}
x \oplus y & =\vee\left(x \wedge a_{\alpha} \oplus y \wedge a_{\alpha}: \alpha \in \Sigma\right) \\
& =\vee\left((x \oplus y) \wedge a_{\alpha}: \alpha \in \Sigma\right) \leq a .
\end{aligned}
$$

Therefore, $[0, a]$ is an ideal. To prove that $a$ is central, we need to prove that $[0, a]$ is a central ideal, i.e. that $[0, a]$ is a Riesz ideal. 
Assume that $z \leq x \oplus y$, where $z \in[0, a], x, y \in E$. Then $z=\oplus\left(z \wedge a_{\alpha}: \alpha \in \Sigma\right)$. For $\forall \alpha \in \Sigma, z \wedge a_{\alpha} \leq x \wedge a_{\alpha} \oplus y \wedge a_{\alpha}$. Put $z_{1}=\oplus\left(x \wedge a_{\alpha}: \alpha \in \Sigma\right), z_{2}=\oplus\left(y \wedge a_{\alpha}\right.$ : $\alpha \in \Sigma)$. Obviously, $z \leq z_{1} \oplus z_{2}, z_{1}, z_{2} \in[0, a]$ and $z_{1} \leq x, z_{2} \leq y$. This proves that $[0, a]$ is a central ideal, i.e. $a$ is central.

Theorem 3.5. Let $E$ be an orthocomplete effect algebra. Then the centre $C(E)$ of $E$ is a complete Boolean algebra. Moreover, all suprema and infima in $C(E)$ coincide with those in $E$.

Proof. Let $\left(a_{\alpha}: \alpha \in \Sigma\right)$ be a family of elements in $C(E)$ indexed by a set $\Sigma$. We will prove that $\vee\left(a_{\alpha}: \alpha \in \Sigma\right)$ exists in $E$ and belongs to $C(E)$. The latter statement is true for any finite set, so we may assume that $\Sigma$ is infinite. Let $\sigma$ be the least ordinal corresponding to $\operatorname{card}(\Sigma)$. We may assume that $\sigma$ is a limit ordinal, and replace the set $\Sigma$ by the set $(\alpha: \alpha<\sigma)$, so that we are dealing with an ordinal-indexed family. Further we proceed by a transfinite induction. Assume that $y_{\alpha}=\vee\left(x_{\rho}: \rho<\alpha\right)$ exists and belongs to $C(E)$ for every $\alpha<\sigma$. This family $\left(y_{\alpha}: \alpha<\sigma\right)$ is nondecreasing, and $\left(y_{\alpha+1} \ominus y_{\alpha}: \alpha+1<\sigma\right)$ is an orthogonal family. Indeed, choose a finite subset $\alpha_{1}<\alpha_{2}<\cdots<\alpha_{n}$ with $\alpha_{n}+1<\sigma$. We then have $y_{\alpha_{1}} \leq y_{\alpha_{1}+1} \leq y_{\alpha_{2}} \leq y_{\alpha_{2}+1} \leq \cdots \leq y_{\alpha_{n}} \leq y_{\alpha_{n}+1}$. Then

$$
\begin{aligned}
& \left(y_{\alpha_{1}+1} \ominus y_{\alpha_{1}}\right) \oplus\left(y_{\alpha_{2}} \ominus y_{\alpha_{1}+1}\right) \oplus \cdots \oplus\left(y_{\alpha_{n}+1} \ominus y_{\alpha_{n}}\right) \\
& \quad=y_{\alpha_{n}+1} \ominus y_{\alpha_{1}} \geq\left(y_{\alpha+1} \ominus y_{\alpha_{1}}\right) \oplus \cdots \oplus\left(y_{\alpha_{n}+1} \ominus y_{\alpha_{n}}\right) .
\end{aligned}
$$

Hence

$$
z=\oplus\left(y_{\alpha+1} \ominus y_{\alpha}: \alpha+1<\sigma\right)
$$

exists and belongs to $C(E)$ by Theorem 3.4. We will prove that $z$ is the desired join $\vee\left(x_{\rho}: \rho<\sigma\right)$.

First, we note that if $z$ is an upper bound of the set $\left(x_{\rho}: \rho<\sigma\right)$, then it is the least one. For if $w \geq x_{\rho}$ for all $\rho<\sigma$, then for all $\alpha+1<\sigma$,

$$
w \geq \vee\left(x_{\rho}: \rho<\alpha+1\right)=y_{\alpha+1} \geq y_{\alpha+1} \ominus y_{\alpha} .
$$

By Lemma 3.3. $z=\vee\left(y_{\alpha+1} \ominus y_{\alpha}: \alpha+1<\rho\right)$, which yields $w \geq z$. Hence it is enough to show that $z \geq x_{\beta}$ for every $\beta<\sigma$.

If $\beta<\sigma, \sigma$ being a limit ordinal, we have $\beta+2<\sigma$, whence

$$
\begin{aligned}
x_{\beta} & \leq \vee\left(x_{\rho}: \rho \leq \beta+1\right)=y_{\beta+1} \\
& =\vee\left(y_{\alpha}: \alpha<\beta+2\right)=\oplus\left(y_{\rho+1} \ominus y_{\rho}: \rho+1<\beta+2\right) \leq z .
\end{aligned}
$$

This proves the theorem.

\section{Orthocomplete And Atomic efFect Algebras}

Recall that an element $a \neq 0$ in an effect algebra $E$ is called an atom if $x \leq a$ implies $x=a$ or $x=0$. An effect algebra $E$ is atomic if every element in $E$ majorizes an atom.

Theorem 4.1. Let $E$ be an orthocomplete effect algebra and let $x \in E$ be an atom. Then $\wedge(z \in C(E): z \geq x)$ is an atom in $C(E)$.

Proof. Put $c(x)=\wedge(z \in C(E): z \geq x)$. By Theorem 3.5 the element $c(x)$ exists and belongs to $C(E)$. To prove that $c(x)$ is an atom of $C(E)$, assume that $d \in C(E)$, $d \leq c(x)$. Then $c(x)=d \oplus\left(d^{\prime} \wedge c(x)\right)$, and

$$
x=x \wedge c(x)=x \wedge d \oplus x \wedge\left(d^{\prime} \wedge c(x)\right) .
$$


If $x \neq d$, then $x \wedge d=0$, because $x$ is an atom, and hence $x \leq d^{\prime} \wedge c(x)$. Since $d^{\prime} \wedge c(x)$ belongs to $C(E)$, we obtain, by the definition of $c(x)$, that $c(x) \leq d^{\prime} \wedge c(x) \leq d^{\prime}$. Then we obtain $d \leq c(x), d \leq c(x)^{\prime}$ hence $d=0$. This concludes the proof.

Lemma 4.2. Let $c$ be a central element of an effect algebra $E$. The centre of the effect algebra $[0, c]_{E}$ consists of elements $z \wedge c, z \in C(E)$.

Proof. Since $c$ is central in $E$, we may write $E \simeq[0, c]_{E} \times\left[0, c^{\prime}\right]_{E}$. If $d$ is central in $[0, c]_{E}$, then $E \simeq[0, d]_{E} \times\left[0, d^{\prime} \wedge c\right]_{E} \times\left[0, c^{\prime}\right]_{E}$, so that $d \in C(E)$. If $z \in C(E)$, then $z \wedge c, z^{\prime} \wedge c$ are orthogonal elements of $C(E)$ with $z \wedge c \oplus z^{\prime} \wedge c=c$, and therefore $[0, c]_{E}=[0, c \wedge z]_{E} \times\left[0, c \wedge z^{\prime}\right]_{E}$, hence $z \wedge c$ is central in $[0, c]_{E}$.

Theorem 4.3. Every orthocomplete atomic effect algebra is a direct product of irreducible effect algebras.

Proof. Since $E$ is atomic, under every element $c$ in the centre $C(E)$ of $E$ there is an atom $x$ of $E$. Theorem 4.1 implies that the element $c(x)=\wedge(z \in C: x \leq z)$ is an atom of $C(E)$. Clearly, $c(x) \leq c$. It follows that $C(E)$ is an atomic Boolean algebra, and by Theorem 3.5 $C(E)$ is complete. Let $\left(c_{\alpha}: \alpha \in \Sigma\right)$ denote the set of all atoms of $C(E)$. Then $\left(c_{\alpha}: \alpha \in \Sigma\right)$ is an orthogonal set, and by Theorem 3.4 we have

$$
E \simeq \prod_{\alpha \in \Sigma}\left[0, c_{\alpha}\right]_{E}
$$

By Lemma 4.2, the centre of $\left[0, c_{\alpha}\right]_{E}$ consists of $\left\{0, c_{\alpha}\right\}$, hence $\left[0, c_{\alpha}\right]_{E}$ is irreducible.

\section{REFERENCES}

1. Bennett, M.K., Foulis, D.J., Phi-symmetric effect algebras, Found. Phys. 25 (1995), 1699-1722. MR 97a:81015

2. Chang, C.C., Algebraic analysis of many-valued logic, Trans. Amer. Math. Soc. 88 (1958), 467-490. MR 20:821

3. Chevalier, G., Pulmannová, S., Some ideal lattices in partial abelian monoids and effect algebras, Order 17 (2000), 75-92. MR 2001g:06004

4. Dvurečenskij, A., Pulmannová, S., New Trends in Quantum Structures, Kluwer Academic Publ., Dordrecht and Ister Science Press, Bratislava, 2000. MR 2002h:81021

5. Foulis, D., Bennett, M.K., Effect algebras and unsharp quantum logics, Found. Phys. 24 (1994), 1331-1352. MR 95k:06020

6. Giuntini, R., Greuling, H., Toward a formal language for unsharp properties, Found. Phys. 19 (1989), 931-945. MR 90j:81017

7. Grätzer, G., Universal algebra, Springer-Verlag, second edition (1979), 80-81. MR 80g:08001

8. Greechie, R., Foulis, D., Pulmannová, S., The center of an effect algebra, Order 12 (1995), 91-106. MR 96c:81026

9. Habil, E., Orthosummable orthoalgebras, Inter. J. Theor. Phys. 33 (1994), 1957-1984. MR 95k:81009

10. Holland, S. Jr., An m-orthocomplete orthomodular lattice is m-complete, Proc. Amer. Math. Soc. 24 (1970), 716-718. MR 41:1604

11. Jenča, G., A Cantor-Bernstein type theorem for effect algebras, Algebra Universalis (to appear).

12. Jenča, G., Subcentral ideals in generalized effect algebras, Inter. J. Theor. Phys. 39 (2000), 745-755. MR 2001j:81018

13. Jenča, G., Pulmannová, S., Quotients of partial abelian monoids and the Riesz decomposition property, Algebra Universalis 47 (2002), 443-477.

14. Kôpka, F., Chovanec, F., D-posets, Math. Slovaca 44 (1994), 21-34. MR 95i:03134

15. Mundici, D., Interpretations of AF $C^{*}$-algebras in Lukasiewicz sentential calculus, J. Funct. Anal. 65 (1986), 15-63. MR 87k:46146 
16. Pulmannová, S., Congruences in partial abelian semigroups, Algebra Universalis 37 (1997), 119-140. MR 97i:06001

17. Pulmannová, S., On connections among some orthomodular structures, Demonstratio Math. 30 (1997), 313-328. MR 98m:06004

18. Schröder, B., On three notions of orthosummability in orthoalgebras, Inter. J. Theor. Phys. 38 (1999), 3305-3313. MR 2001g:81015

19. Riečanová, Z., On proper orthoalgebras, difference posets and Abelian inverse semigroups, Tatra Mt. Math. Publ. 10 (1997), 119-128. MR 98d:06003

Department of Mathematics, Faculty of Electrical Engineering and Information Technology, Ilkovičova 3, 81219 Bratislava, Slovakia

E-mail address: jenca@kmat.elf.stuba.sk

Mathematical Institute, Slovak Academy of Sciences, Štefánikova 49, 81473 BratiSLAVA, SLOVAKIA

E-mail address: pulmann@mat.savba.sk 